clinical trials carried out for dermatological and rheumatic diseases describe exacerbations or new cases of inflammatory bowel disease (IBD), with a low incidence of 0.7 per 100 patient-years in patients with AS. We present a case series of patients who developed new onset severe colitis after the commencement of Secukinumab for the treatment of AS.

Presentation and Diagnosis Clinical and electronic case notes for 3 patients, who developed colitis after loading dose of Secukinumab were reviewed. Histology of endoscopic biopsies was also reviewed specifically for infective and inflammatory colitis. 2 of 3 patients presented with diarrhoea and abdominal pain after loading dose of Secukinumab and one presented with bloody diarrhoea. All three cases were investigated for infective causes with Stool culture, Entamoeba screen and CMV screen.

Results All 3 patients were male and presented from July 2018 to July 2019. The mean age was 57.33 years. One case had a subtotal colectomy for Ulcerative Colitis 10 years ago with pouch formation. 2 patients had received Adalimumab for AS in the past and one was treated with infliximab. No case had evidence of infection. Endoscopic examination for the 2 patients showed severe colitis. The patient with pouch did not have an endoscopy. Histology for the 2 cases confirmed severe active colitis of idiopathic nature. All three cases received steroids and Secukinumab was stopped. The patient with pouchitis responded to oral steroids alone. One of the patients was started on Adalimumab whilst the other received IV steroids and ciclosporin as the initial response to steroids was poor.

Discussion These cases present the association between the initiation of Secukinumab therapy and the development of severe colitis. Though there are no formal guidelines on the management of such cases, our patients responded to other biological and non-biological agents with rapid resolution. Clinicians should be aware of the association between this IL-17 antagonist and the development of severe colitis and prescribers should evaluate individual risk factors prior to its commencement with close monitoring. The pathogenesis behind this is not fully understood and requires further research.

\section{P164 CORRELATION BETWEEN PHYSICIAN AND PATIENT DISEASE ASSESSMENTS IN ULCERATIVE COLITIS: 2- YEAR UK DATA FROM ICONIC}

${ }^{1}$ Neeraj Bhala*, ${ }^{2}$ Ailsa Hart, ${ }^{3}$ David Watts, ${ }^{4}$ Stephen Lewis, ${ }^{5,6}$ Subrata Ghosh, ${ }^{7}$ Stijn van Haaren, ${ }^{7}$ Chris Hansell, ${ }^{8}$ Tariq Ahmad. 'Queen Elizabeth Hospital Birmingham, Birmingham, UK; ${ }^{2} I B D$ Unit, St Mark's Hospital, Harrow, UK; ${ }^{3}$ NHS Forth Valley, Stirling, UK; ${ }^{4}$ Plymouth Hospitals NHS Trust, Plymouth, UK; ${ }^{5}$ NIHR Biomedical Research Centre, University of Birmingham, Birmingham, UK; ${ }^{6}$ University Hospitals Birmingham NHS Foundation Trust, Birmingham, UK; ${ }^{7}$ AbbVie Limited, Maidenhead, UK; ${ }^{8}$ Royal Devon and Exeter Hospital, Exeter, UK

\subsection{6/gutjnl-2020-bsgcampus.239}

Introduction ICONIC is the largest prospective multi-country observational study assessing burden in adult ulcerative colitis (UC) patients under routine care. This local subanalysis evaluated the level of agreement between UK patients and physicians for measures of disease activity over 2 years.

Methods Adults with early UC (diagnosed $\leq 36$ months) were included regardless of disease severity/treatment. Patient self-
Abstract P164 Table 1 Spearman correlation coefficients between instruments

\begin{tabular}{lll}
\hline Variables, $r$ (p-value) & BL & 2-Years \\
\hline SCCAI (physician) vs. P-SCCAI & $0.86(<0.0001) n=62$ & $0.72(<0.0001) n=34$ \\
SCCAI (physician) vs. PRISM(physician) & $-0.64(<0.0001) n=63$ & $-0.64(<0.0001) n=34$ \\
PRISM (patient) vs. PRISM & $0.67(<0.0001) n=63$ & $0.60(0.0004) n=31$ \\
(physician) & & \\
PRISM (patient) vs. SIBDQ & $0.71(<0.0001) n=63$ & $0.74(<0.0001) n=33$ \\
PRISM (patient) vs. PHQ-9 & $-0.56(<0.0001) n=63$ & $-0.48(0.0044) n=33$ \\
PRISM (patient) vs. P-SCCAI & $-0.58(<0.0001) n=62$ & $-0.67(<0.0001) n=33$ \\
\hline
\end{tabular}

assessments of disease activity/impact included: disease severity, Pictorial Representation of Illness and Self-Measure (PRISM, a measure of perceived disease impact; lower scores=greater burden); Patient Health Questionnaire-9 (PHQ-9); Short Inflammatory Bowel Disease Questionnaire (SIBDQ); patientmodified Simple Clinical Colitis Activity Index (P-SCCAI). Physician assessments included: clinical parameters; PRISM; SCCAI. Correlations between measures were assessed using Spearman's correlations.

Results 63 patients were included (59\% female; mean age 43 years; median time since diagnosis 126 days); 98\% patients received treatment post-diagnosis. Physician-assessed baseline (BL) severity was: in remission 16 (25\%), mild 18 (29\%), moderate 18 (29\%), severe 11 (17\%). Overall, 48\% patients agreed with physician-assessed severity (remission 50\%, mild $68 \%$, moderate 28\%, severe 46\%). Table 1 shows correlation coefficients between measures at BL and 2-years. At 2-years, mean \pm SD P-SCCAI/physician SCCAI scores were 2.6 \pm 2.6 and $1.5 \pm 1.5$; patient and physician PRISM scores were 5.2 \pm 2.6 and $5.2 \pm 2.1$.

Conclusion Persistently high UC burden was observed over 2 years, despite treatment. PRISM, used for the first time in UC, was moderately correlated with disease-specific (SIBDQ/ SCCAI) and general depression (PHQ-9) measures. Alignment between patients and physicians on disease activity/severity varied but was greatest for SCCAI.

\section{P165 DISEASE-RELATED WORRIES AND CONCERNS IN UK PATIENTS WITH ULCERATIVE COLITIS: 2-YEAR DATA FROM ICONIC}

${ }^{1}$ Neeraj Bhala*, ${ }^{2}$ Ailsa Hart, ${ }^{3}$ David Watts, ${ }^{4}$ Stephen Lewis, ${ }^{5,6}$ Subrata Ghosh, ${ }^{7}$ Stijn van Haaren, ${ }^{7}$ Chris Hansell, ${ }^{8}$ Tariq Ahmad. ${ }^{1}$ Queen Elizabeth Hospital Birmingham, Birmingham, UK; ${ }^{2} I B D$ Unit, St Mark's Hospital, Harrow, UK; ${ }^{3}$ NHS Forth Valley, Stirling, UKi ${ }^{4}$ Plymouth Hospitals NHS Trust, Plymouth, UK; ${ }^{5}$ NIHR Biomedical Research Centre, University of Birmingham, Birmingham, UK; ${ }^{6}$ University Hospitals Birmingham NHS Foundation Trust, Birmingham, UK; ${ }^{7}$ AbbVie Limited, Maidenhead, UK; ${ }^{8}$ Royal Devon and Exeter Hospital, Exeter, UK

\subsection{6/gutjnl-2020-bsgcampus.240}

Introduction ICONIC is the largest prospective, multi-country observational study assessing cumulative disease-associated burden in adults with ulcerative colitis (UC) under routine care. This local subanalysis evaluated patient worries and concerns over 2 years in UK patients using the Rating Form of Inflammatory Bowel Disease (IBD) Patient Concerns (RFIPC) questionnaire.

Methods Adults with early UC (diagnosed $\leq 36$ months) were included irrespective of treatment regimen/disease 